\title{
Transboundary aquifers, il ruolo dell'idrogeologia regionale
}

\author{
Francesco La Vigna \\ LinQ - Laboratorio di Idrogeologia Numerica e Quantitativa \\ Università degli Studi Roma Tre - Roma \\ lavigna@uniroma3.it
}

Un concetto elementare dell'idrogeologia, che si impara fin dalle prime lezioni sui banchi dell'università, è che il bacino idrogeologico, molto spesso, non corrisponde affatto al bacino idrografico sovraimposto. Questo comporta necessariamente l'esistenza di spartiacque sotterranei che molto spesso non corrispondono a quelli topografici.

I confini politici delle nazioni e quelli amministrativi delle regioni, sono quasi sempre basati sui bacini idrografici e sugli spartiacque o corsi d'acqua, quando addirittura non siano tracciati a tavolino senza seguire motivi almeno geografici.

Dalla combinazione dei concetti precedentemente espressi nasce la definizione di acquifero transfrontaliero, o meglio transboundary aquifer. Questi sono quindi quegli acquiferi che si trovano a cavallo di un confine politico, con una direzione di flusso sotterraneo che può propendere per uno dei due stati, determinando quindi la sua ricarica in uno stato, e il suo immagazzinamento o la sua emergenza nell'altro. Il problema però non è solo quantitativo, ma anche qualitativo: è il caso di acquiferi contaminati che ricevono la contaminazione in uno stato e che la portano seguendo le linee di flusso verso lo stato adiacente.

La gestione e lo studio di questi acquiferi come si può immaginare implicano un'integrazione dell'idrogeologia regionale con discipline come la geopolitica, l'economia e le leggi internazionali (Salman, 2011). Negli ultimi anni, la sicurezza idrica per l'umanità e per l'ambiente naturale ha avuto un ruolo centrale in gran parte del dialogo sulla politica internazionale (Feitelson, 2006, Puri e Aureli, 2005, Scheumann e Alker, 2009). I governi hanno riaffermato la necessità di azioni coordinate per proteggere le popolazioni vulnerabili, e l'ambiente naturale, che si basano su un uso sostenibile delle acque nelle falde acquifere. Le agenzie internazionali che lavorano con le organizzazioni nazionali e regionali hanno messo in evidenza il fatto che molte risorse idriche sotterranee si trovano in contesti transfrontalieri e che per la corretta gestione di queste risorse, la cooperazione tra i paesi è fondamentale. I governi hanno partecipato, su iniziativa dell'UNESCO, all'International Shared (transboundary) Aquifer Resources Management (ISARM) ( www.isarm.org ), facente parte del Programma Idrologico Internazionale (IHP) sempre in seno all'UNESCO. Il programma ISARM, che è operativo dal 2003, beneficia anche di una stretta collaborazione con l'Associazione Internazionale degli idrogeologi (IAH), con l'Organizzazione degli Stati Americani (OAS), con altre istituzioni regionali e internazionali, nonché con le organizzazioni delle Nazioni Unite come l'Organizzazione per l'alimentazione e l'agricoltura (FAO) e dalla Commissione Economica delle Nazioni Unite per l'Europa (UNECE), per citarne solo alcuni. Uno degli obiettivi del programma ISARM è stato quello di effettuare un inventario globale delle falde acquifere transfrontaliere; esse sono state sintetizzate in un atlante che fornisce anche uno stato di fatto della recente risoluzione dell'Assemblea generale delle Nazioni Unite sul diritto degli acquiferi transfrontalieri (Puri e Aureli, 2012).

Lo studio di un acquifero posto al confine tra due stati non comporta, ovviamente, metodologie di studio e di analisi differenti da quelle che vengono normalmente usate nell'idrogeologia regionale classica. La principale sfida tecnica consiste nell'armonizzazione e omogeneizzazione dei dati lungo i confini nazionali. E' infatti lungo questa sezione di acquifero che devono essere molto chiari i rapporti di scambio, l'andamento delle linee di flusso, la presenza di acquiferi confinati e le loro aree di ricarica. Questo per poter produrre un modello concettuale il più possibile realistico, da poter essere poi usato nei rapporti e nelle controversie internazionali.

A livello globale sono molti gli acquiferi transfrontalieri su cui si vi è ampia bibliografia. Si cita ad esempio il Guarany Aquifer System (GAS), condiviso tra Argentina, Uruguay, Paraguay e Brasile, un acquifero che, con i suoi 1,2 milioni di $\mathrm{km}^{2}$ di estensione e le cui risorse idriche sono state stimate in 37 milioni di $\mathrm{km}^{3}$, costituisce il più grande corpo acquifero dell'America latina (Gomez, et al., 2010, Kemper, et al., 2003, Schmidt e Vassolo, 2011) e la risorsa idrica potabile per una popolazione di circa 15 milioni di persone. Altro grande transboundary aquifer è il Nubian Sandstone Aquifer System (NSAS), condiviso tra Chad, Egitto, Libia e Sudan. Questo gigantesco corpo acquifero corrisponde al più grande acquifero fossile a livello mondiale e, data la natura prevalentemente desertica delle nazioni in cui è collocato, costituisce una risorsa d'importanza particolarmente strategica (Nour, 1996, Reynerio Fagundo-Castillo, et al., 2008).

Diversi poi sono i casi in area mediorientale dove molte tensioni sono anche legate a risorse idriche contese come in Israele e Palestina (Feitelson, et al., 2012) e al confine tra Turchia e Siria (Oztan e Axelrod, 2011).

$\mathrm{Nel}$ continente europeo secondo un rapporto della Commissione delle Nazioni Unite per l'Europa (UNECE - che non comprende solo i paesi della Comunità Europea ma anche paesi dell'est e del medio oriente) i corpi d'acqua superficiali e sotterranei transfrontalieri svolgono un ruolo fondamentale per l'area. Questi occupano più del $40 \%$ della superficie europea e asiatica della regione, in cui risiedono circa 460 milioni di abitanti, ossia più del $50 \%$ della popolazione europea $e$ asiatica dell'UNECE. Questi corpi idrici collegano popolazio- 
ni dei vari paesi e la loro gestione ragionevole e sostenibile è fondamentale per il sostentamento delle persone e il benessere in tutta la regione. Pur essendo abbastanza diffusi vi è una forte concentrazione di transboundary aquifers nel sud-est del continente, ed in particolare tra Croazia e Slovenia, tra Croazia e Bosnia, tra Serbia e Ungheria, tra Serbia e Romania e tra Romania e Bulgaria (Horvat e Rubinic, 2006).

L'Italia, ovviamente può presentare questo tipo di problematica solo nel suo tratto non peninsulare. Nel arco alpino nord-occidentale sono prevalenti i casi di corpi idrici superficiali transfrontalieri, mentre nel nord-est ci sono i casi più importanti di transboundary aquifers nazionali, al confine con la Slovenia. Le controversie sulla gestione delle acque sotterranee possono nascere, invece, nel nostro territorio, considerando i limiti amministrativi delle singole regioni. Non a caso infatti il nostro ordinamento prevede i cosiddetti "tribunali delle acque" regionali e nazionale per gestire le controversie in questo ambito.

Considerate le problematiche in atto tra stati che condividono vasti acquiferi, e i possibili conflitti che potrebbero insorgere in visione anche di possibili cambiamenti climatici (Shiva, 2002), il ruolo dell'idrogeologia regionale è sicuramente di primaria importanza nelle attività diplomatiche e di peacekeeping degli organismi internazionali.

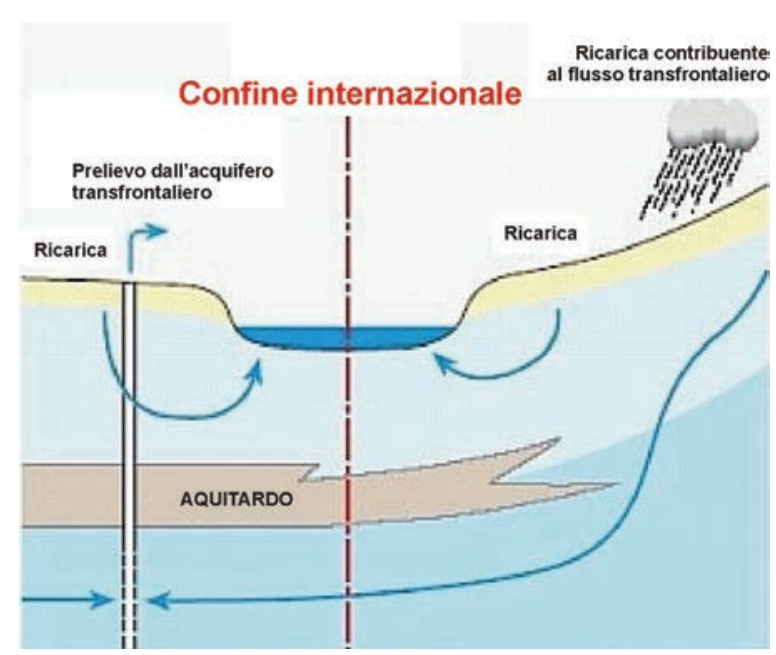

Fig.1: Concettualizzazione di un tipico acquifero transfrontaliero ( modificata da www.isarm.org )

\section{BIBLIOGRAFIA}

Feitelson E (2006) Impediments to the management of shared aquifers: A political economy perspective. Hydrogeology Journal 14: 319329 DOI 10.1007/s10040-005-0481-2

Feitelson E, Tamimi A, Rosenthal G (2012) Climate change and security in the Israeli-Palestinian context. Journal of Peace Research 49: 241-257 DOI 10.1177/0022343311427575

Gomez AA, Rodriguez LB, Vives LS (2010) The Guarani Aquifer System: estimation of recharge along the Uruguay-Brazil border Hydrogeology Journal 18: 1667-1684 DOI 10.1007/s10040-0100630-0

Horvat B, Rubinic J (2006) Annual runoff estimation - an example of karstic aquifers in the transboundary region of Croatia and Slovenia. Hydrological Sciences Journal-Journal Des Sciences Hydrologiques 51: 314-324 DOI 10.1623/hysj.51.2.314

Kemper KE, Mestre E, Amore L (2003) Management of the guarani aquifer system - Moving towards the future. Water International 28: $185-200$

Nour S (1996) Groundwater potential for irrigation in the East Oweinat area, Western Desert, Egypt. Environmental Geology 27: 143-154

Oztan M, Axelrod M (2011) Sustainable transboundary groundwater management under shifting political scenarios: the Ceylanpinar Aquifer and Turkey-Syria relations. Water International 36: 671685 DOI 10.1080/02508060.2011.601546

Puri S, Aureli A (2005) Transboundary aquifers: A global program to assess, evaluate, and develop policy. Ground Water 43: 661-668 DOI 10.1111/j.1745-6584.2005.00100.x

Puri S, Aureli A (2012) ATLAS of transboundary acquifers. Global maps, regional cooperation and local inventories. UNESCO

Reynerio Fagundo-Castillo J, Jose Carrillo-Rivera J, AntigueredadAuzmendi I, Gonzalez-Hernandez P, Pelaez-Diaz R, HernandezDiaz R, Caceres-Govea D, Ramon Hernandez-Santana J, SuarezMunoz M, Melian-Rodriguez C, Rodriguez-Pina M. (2008) Chemical and geological control of spring water in Eastern Guaniguanico mountain range, Pinar del Rio, Cuba. Environmental Geology 55: 247-267 DOI 10.1007/s00254-007-1000-7

Salman MA (2011) The World Bank policy and practice for projects affecting shared aquifers. Water International 36: 595-605 DOI $10.1080 / 02508060.2011 .602925$

Scheumann W, Alker M (2009) Cooperation on Africa's transboundary aquifers-conceptual ideas. Hydrological Sciences JournalJournal Des Sciences Hydrologiques 54: 793-802 DOI 10.1623/ hysj.54.4.793

Schmidt G, Vassolo S (2011) Investigations of a key groundwater system in South America: the Guarani Aquifer in Paraguay. Grundwasser 16: 187-194 DOI 10.1007/s00767-011-0171-z

Shiva V (2002) Water Wars. Privatisation, pollution and profit. South End Press, Cambridge, MA (USA) 